\title{
EFFECT OF COLOURS IN MANUAL DATA TYPING
}

\author{
Melih Kirlidog \\ Department of Computer Engineering, Marmara University, Istanbul, Turkey \\ School of IT, North-West University Vaal Campus, South Africa \\ melihk76@gmail.com
}

\begin{abstract}
Although there is a large body of literature on research into colour in human-computer interaction, the overwhelming majority of the literature emphasises the cognition by computer users. However, colour is also important in this interaction when users manually type data into a computer. This paper investigates the effect of colour combinations on manual data typing. To this end, three experiments were conducted where the subjects were requested to read several texts with different colour combinations and re-type them in the same screen. Typing accuracy and speed is measured as the dependent variable across different colour combinations. Three experiments were conducted as such. In the first experiment, display and input windows were close to each other and in the second one they were located in the opposite ends of the screen. The third experiment was a subset of the first one with reversed foreground and background colours. It was found that different colour combinations had varying effects on data typing performance and proximity of the display and input windows was not a significant factor for typing accuracy in a 17-inch screen. The effect of reversing the foreground and background colours was inconclusive with the colour combinations used.
\end{abstract}

\section{KEYWORDS}

Human-computer interaction (HCI), Colour Combinations, Data Typing, Manual Data Entry

\section{INTRODUCTION}

Human-computer interaction (HCI) can have two directions of information flow, namely from human to computer and vice versa. The flow in the human-to-computer direction can be carried out by several methods using different types of hardware, such as direct manipulation through point-and-click with a mouse, [25] keystrokes on a keyboard and the use of a joystick. The most common type of interaction in the computer-to-human direction is via the computer screen, and the only method employed on the screen is the use of shapes and figures with different colours.

Although there is a large body of literature on the use of colour in computer-to-human interaction using a video display terminal (VDT), this is not true for human-to-computer interaction. In other words, the effect of colour and colour combinations on manual data entry on a chromatic VDT is a neglected area in HCI research. The present article investigates the effect of colour combinations on manual data entry performance. To this end, a series of experiments was David C. Wyld et al. (Eds) : CCSIT, SIPP, AISC, PDCTA, NLP - 2014 pp. 65-76, 2014. (C) CS \& IT-CSCP 2014

DOI : $10.5121 /$ csit.2014.4206 
conducted in which the subjects were requested to read various texts on the screen with different colour combinations and re-type them on the same screen.

Since it is not possible to distinguish the cognitive and psycho-motor functions in the readingand-typing cycle, the "manual data entry performance" in this research involves not only the data typing activity, but also the activity of reading from screen. The colour pairs in the display and input windows are the same in a trial. Thus, a combination of these two activities is measured for different colour pairs where the performance is measured by the accuracy and speed of the data typed. In other words, in effect, what is being measured is not only the isolated data entry activity, but also the cognition performance as an input mechanism to data entry.

\section{COMMON TASKS AND DIRECTIONALITY IN HCI}

An overwhelming majority of HCI research is based on human cognition, and the subjects in this line of research usually act as passive observers who are requested either to find irregularities in a given text or perform a reading activity against time. However, manual data typing into the computer is a more important and common task for many computer users, particularly for manual transaction entry clerks. Unlike the screen reading and observation activity, where most colourbased HCI research has been done, manual data typing is a more complicated activity, because the former is only a subset of the latter. In the manual data typing activity computer users not only have to read from either a computer screen or some other medium such as paper, but they also have to perform the psycho-motor action of typing on a keyboard, an activity which also involves reading the screen and observation per se. In other words, computer-to-human interaction is only a cognitive action whereas human-to-computer interaction is a two-phase action in which the first phase mainly involves cognition and the second phase involves both cognition and psycho-motor activity. The directionality is also different in these two kinds of actions. The former is a unidirectional cognitive activity where the information flows from the screen to the user and the latter is clearly a bidirectional activity.

Unlike the older command line interfaces where the user usually manipulated the computer through a series of typed commands, the modern graphical user interface (GUI) environment offers many convenient methods, including direct manipulation. Currently, direct manipulation of the computer through point-and-click with a mouse is the most common method of interacting with computers. However, although less frequent, manual data entry on a keyboard is also used. This method can still be used for various purposes such as short-cutting the navigation through GUI by experienced users, or for performing manual transactions on forms on the screen. Although in the latter case pointing and clicking can also be used to navigate to a particular field on the screen, the bulk of the action is manual data typing into the relevant fields.

\section{COLOUR COMBINATIONS IN HCI}

Almost all computer screens were monochromatic until a few decades ago. This meant that computer users and purchasing officers in companies had to decide on a fixed foreground and background colour combination of the computer monitor at purchase. The 1990s witnessed two important developments that increased the importance of colour in human-computer interaction. Firstly, chromatic screens became widespread and replaced the monochromatic screens, and secondly, the World Wide Web, which is a highly "colourful" medium, was introduced. The web 
and the related software development tools offered great flexibility to designers not only in the selection of colour, but also in other interface features such as layout, design and aesthetics. Currently almost all computer displays are chromatic and all software development platforms enable developers to use colour effectively. Although chromatic monitors are commonly used both in web-based and non-web-based applications, most of the research on colour-related human-computer interaction has been done on web-based applications, [7], [5], [18] possibly due to the prevalence of the web.

Current research on colour combinations on VDTs can be categorised in three groups [7]. In the first group attempts are made to measure readability through visual search tasks where subjects have to identify misspelled words or to find special words in a paragraph of nonsensical anagrams [16], [17], [12]. In the second group readability is measured in terms of the time required to read a specific text [23], [27]. In the third group visual performance is measured as the percentage of correctly recognised characters or words in a text [24], [10].

Although there is some conflicting evidence in the literature it is generally recommended that extremes of colour combinations such as red-green and red-blue should not be used [21] and rather those colours be used conservatively with a maximum of four colours in a single display [25].

Several authors warn about visual discomfort and poor visual performance resulting from the inappropriate use of colours [13], [22]. Some authors have stressed the importance of colour combinations in reading performance [17], [22], [26] and aesthetics [5], [17], [11].

Shieh and Lin [24] found that colour combination significantly affected not only visual performance but also subjective preference. They also found that a blue foreground and a yellow background resulted in both the best performance and the greatest preference rating, whereas purple on red had the worst rating. It is argued that red and blue are on the opposite ends of the spectrum and their combination on the display unit will strain the muscles surrounding the eye [25], [13]. This strain will result in difficulty in reading. The same is true for yellow on purple and magenta on green.

As stated above, there is a substantial body of research on the colour combinations in the humancomputer interface but there is not much research on the effects of inverted colour combinations on common interface tasks such as reading. In one of the few studies on inverted colour combinations, Nielsen [14] argued that the contrast is the same in an inverted colour scheme, yet "it throws people off a little and slows their reading slightly." A number of studies have found that dark text on light background (positive polarity) has better readability than its inverse combination (negative polarity) [6], [19], [1] while some others have found no significant benefit of positive polarity [17], [12], [5].

Some aspects of retention involving colours have been dealt with in HCI research. Noiwan and Norcio [15] investigated the retention of colours in screen banner graphics in a cross-cultural research. Hall and Hanna [5] found that different combinations of web page text/background colours do not have a statistically significant effect on retention. The cycle of reading a text and re-typing it on the keyboard involves the retention of information in memory. In this case, the information is a sentence or a part of a sentence, and it is investigated whether proximity on the screen affects memory retention. 
It must also be stated that some findings of the HCI research on colours are contradictory. As opposed to the research that found colour combinations having significant effect on text search, perceived aesthetics, and reading, some authors found insignificant effects on these tasks. For example, Pace [16] found no significant difference in text search performance among 24 colour combinations and Radl [19] found that, regardless of the specific colour combination, higher levels of contrast appeared to lead to better readability.

\section{RESEARCH DESIGN}

Data manipulation in this research was accomplished by special-purpose software. The software opened two windows on the screen. The first window displayed an English text in a particular combination of foreground and background colours and the subjects were requested to enter the same text manually in the second window which had the same colour combination (Figure 1).

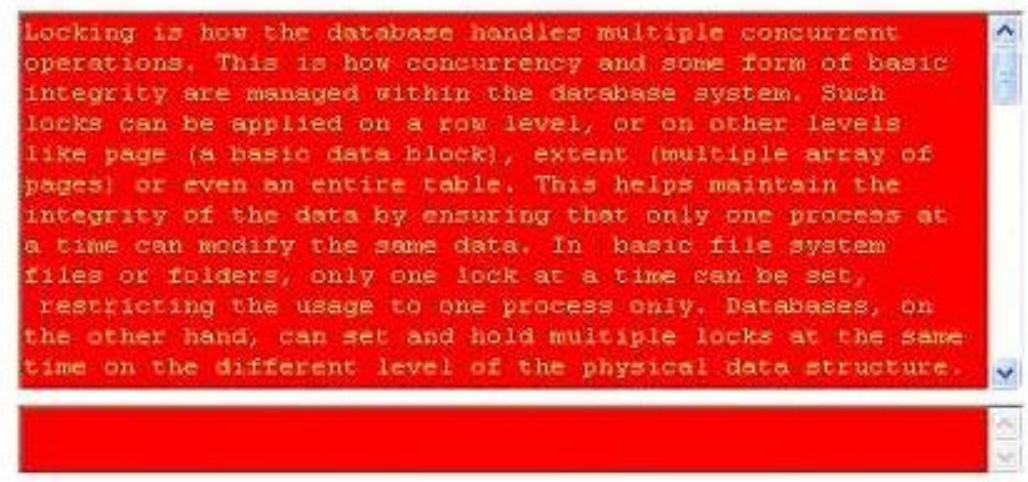

Seconds Remaining: $\quad 64$

Figure 1. Display and entry windows in experiment 1 , trial 1

The displayed texts were stored in the database and the software enabled the database to capture the texts typed by the subjects. The research is a within-subjects design which consisted of 19 trials in three experiments. Each trial had the same text for all subjects. The trials had different colour combinations and different texts of about 400 words each. Hence, a total of 19 texts were involved in the research. In each trial the subjects had 120 seconds for reading the texts in the display window and simultaneously typing them in the entry window. A counter displayed the remaining time during the trial and the software closed both windows automatically after the time had expired.

The performance measured in each trial was the number of correctly typed words in 120 seconds, during which the subjects could type only a certain portion of the given text. In order to familiarise the subjects with the study they were requested to perform an extra trial before starting the actual experiments. The reason for using different texts in the trials was to avoid possible retention of the texts in the subjects' memories across the trials. The texts were selected from an 
introductory computer engineering textbook that was used in an undergraduate course. Since all the subjects had completed that course, they were familiar with the material in the book.

The following rules were applied for calculating the number of correctly typed words:

- Mistyped words were not counted.

- Partially typed words due to time expiry at the end of each trial were not counted.

- In the case of a missing space between two words one of them was not counted, e.g. "dependingon" was counted as one word.

- Lack of a space after a comma or full stop was not counted as an error.

- Missing commas or full stops were not counted as errors.

- Mistyping of lower case and upper case words was not counted as an error.

- A space between words exceeding one character was not counted as an error.

- The words had to be exactly the same as those in the text. For example, typing "subschema" instead of the "sub-schema" in the text was counted as an error.

- Erroneously repeated words were counted as an error.

- A penalty of minus one word was applied in the case of missing a word or block of words.

In the first experiment which contained 13 levels of an independent variable (i.e. colour combination) it was aimed to gauge the typing performance where the two windows were located quite close to each other. In this experiment, the input window was located just under the display window of the text. The distance between the two windows was $0.2 \mathrm{~cm}$ (Fig. 1). The colour combinations used in this experiment are shown in Table 1 with the RGB colour codes.

In order to capture the subjective evaluations of aesthetics and readability, a new window appeared on the screen for data capturing after each trial in the first experiment. The subjects entered two evaluations in this phase through radio buttons in 5-point Likert scale: "I do not like this colour combination" and "It is difficult to read and enter data with this colour combination" (1: completely agree; 5 : completely disagree).

The second experiment aimed to investigate the effect of difference in proximity on typing performance - the two windows were at a distance from each other, i.e. at opposite ends of the screen. The display window was in the upper left corner of the screen and the input window was in the bottom right. The closest distance between the two was $11.5 \mathrm{~cm}$ in this experiment. Three randomly chosen colour pairs used in the first experiment were used in this experiment. Table 5 shows these pairs and descriptive statistics in this experiment.

In the third experiment the aim was to investigate typing performance when the foreground and background colours were reversed. To this end, the reverse of the randomly chosen three colour pairs from the first experiment were used and the screen layout was similar to that of the first experiment, i.e. the windows were $0.2 \mathrm{~cm}$ apart. The colour combinations used in this experiment are shown in Table 7 along with descriptive statistics. 


\subsection{The subjects}

Four female and 26 male computer engineering undergraduate students participated in this research. The average age of the subjects was 23.7, with a standard deviation of 1.53. All the subjects had normal or corrected visual acuity and none of them reported any colour vision deficiencies. Four of the subjects were left-handed. Due to their field of study, the subjects were heavily involved with computers. The starting age at which they became involved ranged from eight to eighteen years, and the mean value for total years of involvement was 9.4 years with a standard deviation of 2.7. Since their medium of instruction is English, the subjects were fluent in both reading and writing in English.

Since the research was mainly based on the performance of fast and accurate data entry, the subjects had to be stimulated for these tasks for the experiment. To this end, better performers were promised additional credits for a course they were attending as well as ten gift books. The experiments were conducted in a computer laboratory. Since the laboratory contained only 20 computers, the experiments were organised in two sessions with two groups of subjects. The subjects were randomly assigned to the groups. The first group comprised 14 students, all of whom were given some credits for participating in the experiment. However, only half of the highest performing subjects (i.e. seven students) were given additional credits. In the same way, only the ten highest performing of the 16 subjects in the other group got the books promised.

\subsection{Software and hardware used}

The VDTs used in the experiments were 17-inch Hewlett-Packard model L1710s which use thin film transistor liquid crystal display (TFT-LCD) technology. The experiments were performed at values of $60 \mathrm{~Hz}$ frequency and 1280 x 1024 pixels resolution. The computers used Microsoft Vista as the operating system and the software was developed using the Microsoft.Net platform. SQL Server 2008 was used for storing the data. The subjects were instructed to use MS Internet Explorer browser instead of Mozilla Firefox which is commonly used in the Department. The reason for this was somewhat paradoxical: Firefox defaults to a higher level of functionality in the context of this experiment by underlining mis-spelled English words. This would have allowed subjects to correct inaccurate entries, which was regarded as "noise" that distorts the real outcome of data entry accuracy.

\section{RESULTS AND DISCUSSION}

\subsection{First experiment (display and input windows in close proximity)}

In this experiment display and input windows are close to each other. The colour pairs used and the mean of correct words for each trial are shown in Table 1 along with other descriptive data such as subjective evaluations of aesthetics and readability. 
Table 1. Descriptive statistics and Tukey HSD test for the first experiment

\begin{tabular}{|c|c|c|c|c|c|c|c|c|c|c|c|}
\hline \multirow{2}{*}{$\begin{array}{l}\text { Tri } \\
\text { al }\end{array}$} & \multirow[b]{2}{*}{$\begin{array}{l}\text { Foreground } \\
\text { (RGB code) }\end{array}$} & \multirow[b]{2}{*}{$\begin{array}{l}\text { Background } \\
\text { (RGB code) }\end{array}$} & \multirow[b]{2}{*}{$\mathbf{N}$} & \multirow[b]{2}{*}{$\begin{array}{l}\text { "Dist } \\
\text { ance" }\end{array}$} & \multicolumn{3}{|c|}{ \# Correct words } & \multicolumn{2}{|c|}{ Aesthetics } & \multicolumn{2}{|c|}{ Readability } \\
\hline & & & & & Mean & St. dev. & $\begin{array}{c}\text { Tukey } \\
\text { group (*) }\end{array}$ & Mean & St. dev. & Mean & $\begin{array}{l}\text { St. } \\
\text { dev. }\end{array}$ \\
\hline 17 & green ( $\# 00$ ffo 0$)$ & orange (\#ffa 500) & 30 & 2 & 58.6 & 15.1 & $\mathrm{C}$ & 3.37 & 0.76 & 3.53 & 0.82 \\
\hline 12 & green $(\# 00 \mathrm{ff} 00)$ & yellow (\#ffffo0) & 30 & 1 & 51.9 & 15.1 & $\mathrm{~B}, \mathrm{C}$ & 3.47 & 0.86 & 3.40 & 0.93 \\
\hline 13 & orange $(\# \mathrm{ffa} 500)$ & blue $(\# 0000 \mathrm{ff})$ & 30 & 3 & 51.9 & 16.2 & $\mathrm{~B}, \mathrm{C}$ & 2.93 & 0.94 & 2.97 & 0.89 \\
\hline 1 & yellow ( $\#$ ffffoo) & red $(\#$ ffo 000$)$ & 30 & 2 & 51.5 & 16.3 & $\mathrm{~B}, \mathrm{C}$ & 2.53 & 1.07 & 2.67 & 0.96 \\
\hline 8 & blue $(\# 0000 \mathrm{ff})$ & yellow (\#ffffo0) & 30 & 2 & 50.6 & 15.7 & $\mathrm{~B}, \mathrm{C}$ & 3.47 & 0.90 & 3.73 & 0.83 \\
\hline 10 & orange ( $\#$ ffa 500$)$ & yellow (\#ffffo0) & 30 & 1 & 49.8 & 15.4 & $\mathrm{~A}, \mathrm{~B}, \mathrm{C}$ & 2.33 & 0.66 & 2.43 & 0.73 \\
\hline 15 & green $(\# 00 \mathrm{ffo} 0)$ & blue $(=0000 \mathrm{ff})$ & 30 & 1 & 49.0 & 14.4 & $A, B, C$ & 1.40 & 0.50 & 1.40 & 0.50 \\
\hline 6 & purple $(\# 800080)$ & $\operatorname{red}(\#$ ffo 000$)$ & 30 & 5 & 48.4 & 14.7 & $A, B, C$ & 1.63 & 0.61 & 1.73 & 0.83 \\
\hline 3 & blue $(\# 0000 \mathrm{ff})$ & $\operatorname{red}(\# \mathrm{ffo} 000)$ & 30 & 4 & 45.3 & 14.5 & A, B & 2.30 & 1.09 & 2.47 & 1.11 \\
\hline 5 & orange $(\# \mathrm{ffa} 500)$ & $\operatorname{red}(\# \mathrm{ffo} 000)$ & 30 & 1 & 43.6 & 15.3 & A, B & 2.00 & 0.83 & 1.97 & 0.72 \\
\hline 7 & green ( $\# 00$ ffo 0$)$ & $\mathrm{red}(\# \mathrm{ff0} 000)$ & 30 & 3 & 42.9 & 11.4 & A, B & 2.17 & 1.12 & 2.30 & 1.12 \\
\hline 16 & purple $(\# 800080)$ & orange (\#ffa 500) & 30 & 4 & 41.6 & 10.0 & A, B & 1.33 & 0.71 & 1.13 & 0.35 \\
\hline 14 & purple $(\# 800080)$ & blue ( $\# 0000 \mathrm{ff})$ & 30 & 1 & 38.2 & 11.9 & $\mathrm{~A}$ & 2.73 & 0.94 & 2.57 & 0.97 \\
\hline
\end{tabular}

(*) Combinations with the same letter are not significantly different among themselves

The table is sorted in descending order of the mean of the number of correct words column. The subjects scored highest in the green-on-orange trial and lowest in the purple-on-blue.

In order to investigate the effect of colour combinations on the speed and accuracy of data entry, an ANOVA test (analysis of variance) was performed where colour combinations were the factors, i.e. independent variables, and correct words constituted the dependent variable. The group sizes were equal, which satisfied the requirement of the ANOVA test. The Shapiro-Wilk test for normality was conducted on the data and it revealed that the distribution was normal only for experiments 12,13 and 17 at $\alpha=0.05$. The data in the other experiments did not have a normal distribution. Nevertheless, the ANOVA test was conducted since the robustness of the test affected by normality is a matter of degree [20], [8]. Prior to the ANOVA test, Levene's test was performed to determine the homogeneity of variances. The test revealed that variances for the groups were not significantly different (Levene statistics $0.782 ; \mathrm{p}=0.669$ ), and thus the assumption for ANOVA was satisfied. The ANOVA test result was $F(12,377)=4.280, p=$ 0.000; which meant that the null hypothesis could be rejected and that there were statistically significant performance differences for manual data entry for different colour combinations. Since the group variances and sample sizes were homogeneous, a Tukey HSD post-hoc test was done to determine the statistically significant different groups among the colour combinations.

The argument about the difficulty of human processing for colour pairs that are distant from each other [25], [13] was also tested with the data of the first experiment. To this end, each colour used in the experiment was given a number that corresponds to its wavelength. Table 2 shows the approximate wavelengths of each colour used in this study and the corresponding number used for an approximate calculation within colour pairs. The "distances" within colour pairs in the trials were calculated as the absolute value of the differences of these numbers and they are shown in Table 1. 
Table 2. Approximate colour wavelengths in the spectrum and the number used for calculating the "distance" within colour pairs

\begin{tabular}{|l|c|c|}
\hline Colour & Wavelength $(\mathbf{n m})$ & Number in the scale \\
\hline Infrared & $>1000$ & \\
\hline Red & 670 & 6 \\
\hline Orange & 610 & 5 \\
\hline Yellow & 575 & 4 \\
\hline Green & 530 & 3 \\
\hline Blue & 470 & 2 \\
\hline Purple & 430 & 1 \\
\hline Ultraviolet & $<300$ & \\
\hline
\end{tabular}

Table 3 shows the Pearson's correlations between the distances and other parameters. Although there was no significant correlation between wavelength distances in colour pairs and correct words, the correlation between the distances and perceived aesthetics was significant. This was also true for distances and readability. Thus, this result partially supports the argument that distant colours are not suitable for human processing. Table 3 also shows that although the correlation between the subjective evaluations of aesthetics and readability is quite high, it is low between correct words and perceived aesthetics. The same is true for correct words and readability.

Table 3. Pearson's correlations for dependent variables in the first experiment $(n=390)$

\begin{tabular}{|l|c|c|c|c|}
\hline & $\begin{array}{c}\text { Mean } \\
\text { (\# correct } \\
\text { words) }\end{array}$ & $\begin{array}{c}\text { Perceived } \\
\text { aesthetics }\end{array}$ & Readability & $\begin{array}{c}\text { Distance in } \\
\text { spectrum }\end{array}$ \\
\hline Mean (\# correct words) & & $0.112^{*}$ & $0.205^{* *}$ & -0.040 \\
\hline Perceived aesthetics & & & $0.813^{* *}$ & $-0.231^{* *}$ \\
\hline Readability & & & & $-0.207^{* *}$ \\
\hline Distance in spectrum & & & & \\
\hline
\end{tabular}

$* \mathrm{p}<0.05$

$* * \mathrm{p}<0.01$

Although a MANOVA test for investigating the relationships between colour combinations and the dependent variables of correct words, perceived aesthetics and readability would be desirable, such a test would be meaningful only if the correlations were moderate [9]. Hence, instead of MANOVA, two separate ANOVA tests were performed where colour combinations were the factors in both tests and perceived aesthetics and readability were the dependent variables.

Table 4. Test for homogeneity of variances for aesthetics and readability in the first experiment

\begin{tabular}{|c|c|c|c|c|}
\hline & Levene's test & df1 & df2 & Significance \\
\hline Aesthetics & 3.007 & 12 & 377 & 0.000 \\
\hline Readability & 5.394 & 12 & 377 & 0.000 \\
\hline
\end{tabular}

As shown in Table 4, Levene's test indicated that the variances were not homogenous both for perceived aesthetics and readability. Therefore Welch and Brown-Forsythe tests were performed for determining whether there were statistically significant differences in perceived aesthetics and readability across different colour combinations. The significance of Welch and Brown-Forsythe tests both for perceived aesthetics and readability was equal to 0.000 , which meant that the null 
hypothesis could be rejected for both. Hence, the differences were significant. For perceived aesthetics $\mathrm{F}(12,377)=21.85, \mathrm{p}<0.05$, and for readability $\mathrm{F}(12,377)=26.27, \mathrm{p}<0.05$.

\subsection{Second experiment (display and input windows are distant from each other)}

Some of the colour pairs that were used in the first experiment were also used in the second experiment. As stated, the aim in this experiment was to gauge the extent to which the proximity of the two windows affected the speed and accuracy of data entry. To this end, results of the second experiment were compared with the first experiment results for the same data sets. The colour pairs used in this experiment are shown in Table 5 along with the descriptive statistics. The pairs are sorted according to the mean of the correct words.

Table 5. Descriptive statistics for the second experiment (windows distant from each other)

\begin{tabular}{|c|c|c|c|c|c|c|}
\hline Trial & Foreground & Background & $\begin{array}{c}\text { Corresponding trial in } \\
\text { experiment 1 }\end{array}$ & $\mathbf{N}$ & $\begin{array}{c}\text { Mean } \\
\text { (\# correct words) }\end{array}$ & St. dev. \\
\hline 2 & yellow & red & 1 & 30 & 50.0 & 12.8 \\
\hline 4 & blue & red & 3 & 30 & 46.1 & 10.6 \\
\hline 9 & blue & yellow & 8 & 30 & 44.0 & 12.0 \\
\hline
\end{tabular}

Since there were only three sets of data for which we wished to gauge the difference, three t-tests were performed instead of ANOVA. The results of these tests are shown in Table 6.

Table 6. t-test for varying proximity $(\mathrm{p}<0.05)$

\begin{tabular}{|l|c|c|c|c|c|}
\hline & \multicolumn{2}{|c|}{ Levene's test } & \multicolumn{4}{c|}{ t-test } \\
\hline Trial pair (foreground, background) & F & Sig. & df & t & Sig. (2-tailed) \\
\hline 1-2 (yellow, red) & 0.358 & 0.552 & 58 & 0.389 & 0.699 \\
\hline 3-4 (blue, red) & 1.379 & 0.245 & 58 & -0.234 & 0.816 \\
\hline 8-9 (blue, yellow) & 0.680 & 0.413 & 58 & 1.831 & 0.072 \\
\hline
\end{tabular}

Higher significance values of Levene's test indicated that the variability of each group was approximately equal, satisfying the requirement for t-tests. This was true for all three experiments.

The t-tests indicated that, contrary to what one would expect, there were no statistically significant differences of typing speed and accuracy arising from the difference in proximity on the screen.

\subsection{Third experiment (foreground and background colours are reversed)}

In the third experiment the aim was to gauge the difference by reversing the foreground and background colours. For this experiment some of the colour pairs in the first experiment were also used. Descriptive data which are sorted according to the mean of the correct words in this experiment are shown in Table 7: 
Table 7. Descriptive statistics for the third experiment (reversed foreground and background colours)

\begin{tabular}{|c|c|c|c|c|c|c|}
\hline Trial & Foreground & Background & $\begin{array}{c}\text { Corresponding trial in } \\
\text { experiment 1 }\end{array}$ & $\mathbf{N}$ & $\begin{array}{c}\text { Mean } \\
\text { (\# correct words) }\end{array}$ & $\begin{array}{c}\text { St. } \\
\text { dev } \\
\text {. }\end{array}$ \\
\hline 20 & red & yellow & 1 & 30 & 53.1 & 15.0 \\
\hline 18 & red & blue & 3 & 30 & 50.1 & 17.7 \\
\hline 19 & yellow & blue & 8 & 30 & 42.5 & 11.1 \\
\hline
\end{tabular}

Three t-tests for three pairs of colour combinations were performed for this experiment. The tests were performed for pairs in the first experiment and the third one. The results are shown in Table 8:

Table 8. t-test for reversed foreground and background colours $(\mathrm{p}<0.05)$

\begin{tabular}{|l|c|c|c|c|c|}
\hline & \multicolumn{2}{|c|}{ Levene's test } & \multicolumn{3}{c|}{ t-test } \\
\hline Trial pair (foreground, background) & F & Sig. & df & T & Sig. (2-tailed) \\
\hline 18-3 (red, blue) - (blue, red) & 1.085 & 0.302 & 58 & 1.141 & 0.259 \\
\hline $19-8$ (yellow, blue) - (blue, yellow) & 2.053 & 0.157 & 58 & -2.320 & 0.024 \\
\hline 20-1 (red, yellow) - (yellow, red) & 0.001 & 0.972 & 58 & 0.404 & 0.688 \\
\hline
\end{tabular}

Levene's tests indicated that variances were homogeneous for all tests. The t-tests revealed that there was no statistically significant difference for the blue-red and yellow-red combinations, but the difference for the blue-yellow combination was significant.

\section{CONCLUSION, LIMITATIONS AND FUTURE WORK}

The investigation in this research was based on three HCI concepts, namely colour combinations, visual performance and manual data entry. Manual data entry performance is measured in terms of correct words entered in read-and-type cycles. Subjective considerations of colour combinations on perceived aesthetics and ease of readability and data entry were also evaluated.

It was found that different colour combinations had varying effects on the performance of manual data entry and that these effects were statistically significant. This is in conformity the majority of earlier research. There were also significant differences on subjective evaluations for aesthetics and readability of colour combinations.

Human memory is heavily involved in the read-and-type cycle. The cycle involves mainly three steps, namely visual cognition, temporary storage of data in human memory, and the typing process. When all other independent variables are kept constant, difference in length between the display and input windows can be expected to lead to decrease in performance, because larger distance can lead to increase in the cycle time which in turn can slow down the process. Nevertheless, it was found that the effect of proximity on a 17-inch screen in a read-and-type cycle was insignificant when the display and the typing windows had the same colour combination.

The effect on performance of reversing the foreground and background colours was somewhat inconclusive. The two trials in this experiment showed insignificant differences, but the difference in one trial was significant. This can be due to the fact that there is indeed a pattern that 
the read-and-type process is affected by reversing some colour pairs and not affected by reversing some others. This can be the topic of further research that involves more colour combinations.

There are some limitations on these findings which mainly stem from the implementation of the RGB colour system in VDTs. While different intensities of the primary colours red, green and blue produce different colours, those colours are also represented in the HSV (hue, saturation, value) colour model where the hue is the dominant wavelength or colour and the value is luminance or brightness. The saturation is the colour intensity spanning from "colourless" or grey to the maximum "colourfulness." These parameters are hardware-dependent and different hardware may produce slightly different colours for the same HSV and RGB codes. Fukuzumi et al. [3] argue that the hue spans various levels of saturation and occupies a large area in a chromaticity coordinate diagram. Hence, a particular colour such as green can take various forms. It is even more difficult to determine the appearance of a particular colour on VDTs due to the hardware difference where even different models of the same manufacturer may produce slightly different results [4]. Additionally, factors such as display resolution in different multimedia devices may lead to slight differences in colour perception [5]. However, these limitations are not applicable for the second and third experiments in this research.

This research is introductory in this specific area of HCI, and further research needs to be done to address its limitations. Such research should also contain other colour combinations that were not used in this research. This has important implications for practice, because as stated before, manual data typing in a "colourful" environment is a quite common computer task in today's world.

\section{REFERENCES}

[1] Bauer, D. \& Cavonius, C.R. (1980). Improving the legibility of visual display units through contrast reversal. In: E. Grandjean \& E. Vigliani (Eds.) Ergonomic aspects of visual display terminals (pp. 137-142) London: Taylor \& Francis.

[2] Radl, G.W. (1980). Experimental investigations for optimal presentation-mode and colours of symbols on the CRT-screen. In E. Grandjean \& E. Vigliani (Eds.), Ergonomic aspects of visual display terminals (pp. 127-136) London: Taylor \& Francis.

[3] Fukuzumi, S., Yamazaki, T., Kamijo, K., \& Hayashi, Y. (1998). Physiological and psychological evaluation for visual display colour readability: A visual evoked potential study and a subjective evaluation study. Ergonomics, 41(1), 89-108.

[4] Gnambs, T., Appel, M., \& Batinic, B. (2010). Color red in web-based knowledge testing. Computers in Human Behavior, 26(6), 1625-1631.

[5] Hall, R. \& Hanna, P. (2004). The impact of web page text-background colour combinations on readability, retention, aesthetics and behavioural intention. Behaviour \& Information Technology, 23(3), 183-195.

[6] Hill, A.L. \& Scharff, L.V. (1997). Readability of screen displays with various foreground/background color combinations, font styles, and font types. In Proceedings of the 11th National Conference on Undergraduate Research (pp. 742 - 746) Austin, TX.

[7] Humar, I., Gradisar, M., \& Turk, T. (2008). The impact of color combinations on the legibility of a web page text presented on CRT displays. International Journal of Industrial Ergonomics, 38(11-12), 885-899.

[8] Kong, S.C. \& Kwok, L.F. (2005). A cognitive tool for teaching the addition/subtraction of common fractions: a model of affordances. Computers \& Education, 45(2), 245 - 265.

[9] Leech, N.L., Barrett, K.C., \& Morgan, G.A. (2005). SPSS for intermediate statistics, 2nd ed. Mahwah, NJ: Lawrence Erlbaum Associates, Inc. 
[10] Lin, C.C. \& Huang, K.C. (2006). Effects of ambient illumination and screen luminance combination on character identification performance of desktop TFT-LCD monitors. International Journal of Industrial Ergonomics, 36(3), 211-218.

[11] Lindgaard, G. (2007). Aesthetics, visual appeal, usability and user satisfaction: what do the user's eyes tell the user's brain? Australian Journal of Emerging Technologies and Society, 5(1), 1-14.

[12] Ling, J. \& van Schaik, P. (2002). The effect of text and background colour on visual search of web pages. Displays, 23(5), 223-230.

[13] Matthews, M.L. (1987). The influence of colour on CRT reading performance and subjective comfort under operational conditions. Applied Ergonomics, 18(4), 323-328.

[14] Nielsen, J. (2000). Designing web usability: the practice of simplicity. Indianapolis, IN: New Riders Publishing.

[15] Noiwan, J. \& Norcio, A.F. (2006). Cultural differences on attention and perceived usability: investigating color combinations of animated graphics. International Journal of Human-Computer Studies, 64(2), 103-122.

[16] Pace, B.J. (1984). Color combinations and contrast reversals on visual display units. In Proceedings of the Human Factors Society 28th Annual Meeting (pp. 326-330) Santa Monica, CA.

[17] Pastoor, S. (1990). Legibility and subjective preference for color combinations in text. Human Factors, 32(2), 157-171.

[18] Pearson, R. \& van Schaik, P. (2003). The effect of spatial layout and link colour in web pages on performance in a visual search task and an interactive search task. International Journal of HumanComputer Studies, 59(3), 327-353.

[19] Radl, G.W. (1980). Experimental investigations for optimal presentation-mode and colours of symbols on the CRT-screen. In E. Grandjean \& E. Vigliani (Eds.), Ergonomic aspects of visual display terminals (pp. 127-136) London: Taylor \& Francis.

[20] Rutherford, A. (2001). Introducing ANOVA and ANCOVA: a GLM approach. London: Sage Publications.

[21] Sanders, M.S. \& McCormick, E.J. (1993). Human factors in engineering and design. Singapore: McGraw-Hill.

[22] Shieh, K.K. \& Chen, M.T. (1997). Effects of screen color combination, work-break schedule, and workplace on VDT viewing distance. International Journal of Industrial Ergonomics, 20(1), 11-18.

[23] Shieh, K.K., Chen, M.T., \& Chuang, J.H. (1997). Effects of color combination and typography on identification of characters briefly presented on VDTs. International Journal of Human Computer Interaction, 9(2), 169-181.

[24] Shieh, K.K. \& Lin, C.C. (2000). Effects of screen type, ambient illumination, and colour combination on VDT visual performance and subjective preference. International Journal of Industrial Ergonomics, 26(5), 527-536.

[25] Shneiderman, B. \& Plaisant, C. (2005). Designing the user interface, 4th ed. Boston, MA: PearsonAddison Wesley.

[26] Wang, A.H. \& Chen, C.H. (2003). Effects of screen type, Chinese typography, text/background color combination, speed, and jump length for VDT leading display on users' reading performance. International Journal of Industrial Ergonomics, 31(4), 249-261.

[27] Wu, J.H. \& Yuan, Y.F. (2003). Improving searching and reading performance: the effect of highlighting and text color coding. Information \& Management, 40(7), 617-637.

\section{AUTHOR}

Melih Kirlidog has BSc degree from Middle East Technical University, Turkey and MBA (in MIS) and PhD degrees from University of Wollongong, Australia. He has worked in the ICT industry in Turkey and Australia for over twenty years. Currently he is working as a faculty member in Marmara University, Turkey.

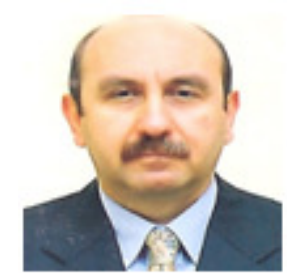

